\title{
Correlation between inflammatory biomarkers and metabolic disorders in HIV infected patients undergoing antiretroviral therapy
}

\author{
Raluca Mihăilescu ${ }^{1 *}$, Victoria Aramă1,2, Cătălin Tilişcan ${ }^{1,2}$, Daniela Munteanu', Viorica Leoveanu', \\ Mihaela Rădulescu ${ }^{1,2}$, Adriana Hristea ${ }^{1,2}$, Cristina Popescu ${ }^{1,2}$, Ruxandra Moroti ${ }^{1,2}$, Violeta Molagic ${ }^{1}$, Raluca Năstase ${ }^{1}$, \\ Loredana Benea', Ana Maria Tudor ${ }^{1,2}$, Mihai Lazăr ${ }^{1,2}$, Anca-Ruxandra Negru', Irina Lăpădat ${ }^{1}$, Ligia Ionescu', \\ Mirela Cernat ${ }^{1}$, Georgeta Jugănaru', Doina Cristea', Adriana Manea', Adrian Streinu-Cercel ${ }^{1,2}$, Daniela Adriana Ion², \\ Sorin Ștefan Aramă²
}

From The 9th Edition of the Scientific Days of the National Institute for Infectious Diseases Prof Dr Matei Bals Bucharest, Romania. 23-25 October 2013

\section{Background}

The objective of the present study was to evaluate the correlation between inflammatory biomarkers and metabolic syndrome and insulin-resistance in HIV seropositive patients, undergoing antiretroviral therapy (cART).

\section{Methods}

We performed a cross-sectional study, conducted as part of the research grant PNCDI2 no.62077/2008 on HIV-infected patients undergoing cART, recruited in a tertiary care hospital, between 2009-2011. BioSource EASIA (Enzyme Amplified Sensitivity Immunoassay) quantified tumor necrosis factor-alpha (TNF alpha), interleukin-6 (IL6), monocyte chemotactic protein 1 (MCP1), high-sensitivity C-reactive protein (hsCRP).

\section{Results}

We included 101 patients, characterized by: M:F ratio 1.3; median age of 31 years. Undetectable HIV viremia was found in $75.2 \%$ of the subjects. The detectable viremia group had median of age 20 years versus 31, CD4 334/cmm versus $546 / \mathrm{cmm}(\mathrm{p}=0.007)$ and 4 therapeutic combinations versus 1 in undetectable patients $(\mathrm{p}=0.000)$. Pathological values of MCP 1 were found 2 times more frequently in the detectable group. Prevalence of metabolic syndrome was 12 versus $18.4 \%$ while prevalence of insulin-resistance was

\footnotetext{
* Correspondence: ralsan@gmail.com

'National Institute for Infectious Diseases "Prof. Dr. Matei Balş", Bucharest, Romania

Full list of author information is available at the end of the article
}

50 versus $64.8 \%$ in detectable versus undetectable patients, $\mathrm{p}>0.05$. Each logarithmical increase of MCP 1 augments the risk of metabolic syndrome by 3.4 times (CI95\% 1.2-10, $\mathrm{p}=0.03$ ) and the risk of insulin-resistance by 2.9 times (CI95\% 1.2-7.7, $\mathrm{p}=0.03)$.

\section{Conclusion}

The prevalence of metabolic syndrome and insulin-resistance are moderate to high. They seem higher in the undetectable group than in the detectable one, probably because of the one-decade difference of age and of the preponderance of overweight males in the undetectable group. Metabolic disorders are not related to viral replication, but probably cART impacts on adipocytes in all patients. MCP 1 is correlated with the presence of both metabolic syndrome and insulin-resistance.

\section{Authors' details}

'National Institute for Infectious Diseases "Prof. Dr. Matei Balş", Bucharest, Romania. ${ }^{2}$ Carol Davila University of Medicine and Pharmacy, Bucharest, Romania.

Published: 16 December 2013

doi:10.1186/1471-2334-13-S1-033

Cite this article as: Mihăilescu et al:: Correlation between inflammatory biomarkers and metabolic disorders in HIV infected patients undergoing antiretroviral therapy. BMC Infectious Diseases 2013 13(Suppl 1):O33. 